Received: 2016.11 .14

Accepted: 2016.11 .30

Published: 2017.08.23

\section{Inter-Observer Agreement of Whole-Body Computed Tomography in Staging and Response Assessment in Lymphoma: The Lugano Classification}

\author{
Ahmed Abdel Khalek Abdel Razek ${ }^{1 \mathrm{ABCDEF}}$, Sameh Shamaa ${ }^{2 \mathrm{CDB}}$, \\ Mahmoud Abdel Lattif ${ }^{\text {BCDE }}$, Hanan Hamid Yousef ${ }^{1 \text { BCD }}$ \\ ${ }^{1}$ Department of Diagnostic Radiology, Mansoura Faculty of Medicine, Mansoura, Egypt \\ 2 Department of Medical Oncology, Mansoura Oncology Center, Mansoura Faculty of Medicine, Mansoura
}

Author's address: Ahmed Abdel Khalek Abdel Razek, Department of Diagnostic Radiology, Mansoura Faculty of Medicine, Mansoura, DK, Egypt, 35512, e-mail: arazek@mans.edu.eg

\title{
Summary
}

Background: to assess inter-observer agreement of whole-body computed tomography (WBCT) in staging and response assessment in lymphoma according to the Lugano classification.

Material/Methods:

Retrospective analysis was conducted of 115 consecutive patients with lymphomas (45 females, 70 males; mean age of 46 years). Patients underwent WBCT with a 64 multi-detector CT device for staging and response assessment after a complete course of chemotherapy. Image analysis was performed by 2 reviewers according to the Lugano classification for staging and response assessment.

Results: The overall inter-observer agreement of WBCT in staging of lymphoma was excellent $(k=0.90$, percent agreement $=94.9 \%)$. There was an excellent inter-observer agreement for stage I $(k=0.93$, percent agreement $=96.4 \%)$, stage II $(k=0.90$, percent agreement $=94.8 \%)$, stage III $(k=0.89$, percent agreement $=94.6 \%)$ and stage IV $(k=0.88$, percent agreement $=94 \%)$. The overall inter-observer agreement in response assessment after a completer course of treatment was excellent $(k=0.91$, percent agreement $=95.8 \%$ ). There was an excellent inter-observer agreement in progressive disease $(k=0.94$, percent agreement $=97.1 \%)$, stable disease $(k=0.90$, percent agreement $=95 \%)$, partial response $(k=0.96$, percent agreement $=98.1 \%)$ and complete response $(k=0.87$, Percent agreement $=93.3 \%$ )

Conclusions: $\quad$ We concluded that WBCT is a reliable and reproducible imaging modality for staging and treatment assessment in lymphoma according to the Lugano classification.

MeSH Keywords: $\quad$ Hodgkin Disease • Neoplasm Staging • Response Elements

PDF file: $\quad$ http://www.polradiol.com/abstract/index/idArt/902370

\section{Background}

Lymphomas, both Hodgkin lymphomas and non-Hodgkin lymphomas, comprise approximately $5 \%$ to $6 \%$ of all malignancies, approximately half of all newly diagnosed hematological tumors and are the fifth most frequently occurring type of cancer in the United States [1,2]. Optimal lymphoma management requires accurate pretreatment staging and reliable assessment of response during and after therapy $[2,3]$. Staging is used to define the anatomic distribution of the disease for purposes of prognosis and treatment planning. Response to treatment serves as an important surrogate for other measures of clinical benefit such as progression-free survival and overall survival. Response is also an important guide in decisions regarding continuation or change of therapy [2-6]. Different classification systems are applied for staging and follow-up of lymphomas. The Lugano classification is the most recent classification system used for staging and treatment response assessment in lymphomas [4-6]. 
Cross-sectional structural and functional imaging plays a crucial role in staging and response assessment in lymphomas. Whole-body imaging plays an essential role in the management of lymphomas, allowing for an evaluation of the full extent of disease at baseline, accurate staging, adaptation of treatment strategies, assessment of treatment response and detection of relapses [7-10]. Positron emission tomography/computed tomography with [18F]-fluorodeoxyglucose (FDG-PET/CT) is commonly used for response assessment in lymphomas, but it is associated with radiation exposure, considerable cost, limited access, and FDG is also taken up at sites of infection and inflammation, which is common in lymphoma patients [11-13]. Whole-body diffusion-weighted MR imaging was used to assess lymphomas, but the examination is time-consuming, expensive and may miss small lesions [14-19]. Whole-body PET-MR imaging has been recently applied for assessments in oncology, but it is not routinely available and still under trial [20]. Whole-body computed tomography (WBCT) was used in oncology and trauma patients [21-22]. Few studies discuss the role of routine contrast-enhanced CT for staging and follow-up of patients with lymphomas [23-25].

\section{Aim of the work}

To assess inter-observer agreement of WBCT in staging and treatment response assessment in lymphomas according to the Lugano classification.

\section{Material and Methods}

Institutional review board approval was obtained and informed consent from the patients was waived because this was a retrospective study. A retrospective study was conducted in 121 patients with lymphomas who underwent WBCT. We included adult patients, aged 18 years or older, who were clinically scheduled to undergo CT for staging of newly diagnosed (histologically proven) Hodgkin or non-Hodgkin lymphomas. We excluded 6 patients from the study, because these patients had undergone CT without contrast medium due to renal impairment. Finally, we included 115 patients (70 males and 45 females, aged 18 to 88 years). The patients presented with fever, pain, weight loss, easy fatigability, swellings, constipation, abdominal enlargement, dysphagia, hoarseness of voice, cough, vomiting, pallor and night sweating. Lymphoma subtypes were diagnosed based on tissue samples obtained by biopsy or surgery, according to the criteria of the current WHO classification of hematological and lymphoid malignancies [26]. Patients with Hodgkin lymphomas received chemotherapy (ABVD - adriamycine, bleomycine, vinblastine, decarbazine) and patients with non-Hodgkin lymphomas received CHOP (cyclophosphamide, adriamycine, oncovin and prednisone). Follow-up WBCT examinations were performed in 103 patients after a complete course of chemotherapy.

All patients were examined with a 64-MDCT scanner (Brilliance 64, Philips Medical Systems Cleveland, $\mathrm{OH}$, USA). All patients were instructed to drink 1-1.5 L of water as oral contrast 30-60 minutes prior to examination. Intravenous injection of non-ionic contrast media (Ultravist $300 \mathrm{mg} / \mathrm{ml}$ ) at a dose of $1.5 \mathrm{ml} / \mathrm{kg}$, via a power injector
(Envision CT injector, MEDRAD, Pittsburgh, PA, USA), with a 20-G intravenous cannula placed in the antecubital fossa was performed at a rate of $3-5 \mathrm{ml} / \mathrm{sec}$. CT examinations were performed in the supine position with feet first. Scanning started 30-60 seconds after contrast injection, extended from the roof of the skull down to the symphysis pubis. A collimation of $64 \times 0.6 \mathrm{~mm}$ with 0.5 second rotation time and a pitch factor of 1.4 were chosen. The tube voltage was $120 \mathrm{kV}, 100-200 \mathrm{mAs}$. All images were reconstructed with an overlapping technique in the axial plane with an effective slice thickness of $1-1.5 \mathrm{~mm}$ and a reconstruction increment of 0.7-0.8 mm. Multi-planar images in the sagittal and coronal planes with section thickness of $4 \mathrm{~mm}$ were reconstructed. Baseline WBCT was performed 2-12 days (mean of 5 days) before initiation of the first cycle of chemotherapy and response assessment was performed 5-15 days (mean of 8 days) after completion of a full course of chemotherapy.

Image analysis was performed by 2 radiologists (AA, MA) with experience of 20 and 10 years in CT scan imaging, respectively. They were blinded to clinical findings and pathological diagnosis. They independently reviewed the images according to the Lugano classification for staging and response assessment in lymphomas [6]. The image analysis was performed for nodal and extra-nodal involvement in different regions of the body using PACS (picture archiving and communication system) for all patients. Staging was done based on the extent of involvement of nodal groups, as follows: stage I, single lymph node group; stage II, multiple lymph node groups ipsilateral to the diaphragm; stage III, involvement of lymph node groups both above and below the diaphragm; and stage IV, noncontiguous extra nodal involvement (e.g., liver, lung, or bone marrow) [6]. The Lugano criteria for response assessment using CT were: (a) complete radiologic response, all nodes smaller than or equal to $1.5 \mathrm{~cm}$ in the longest diameter, disappearance of all CT findings of lymphoma; (b) partial remission, $50 \%$ or greater decrease in disease burden; (c) stable disease, less than $50 \%$ decrease in disease burden; and (d) progressive disease, new or increased adenopathy or new extra-nodal lymphoma [6].

The statistical analysis of data was done by with the Statistical Package for Social Science,version 20 (SPSS Inc., Chicago, Ill, USA). The inter-observer agreement was expressed as a kappa (k) statistic with 95\% confidence intervals (CI) and P value $<0.05$ was considered to indicate statistical significance. The $\kappa$ coefficient is the amount of observed agreement. A $\kappa$ of 0.0 represents agreement that is equal to chance, a $\kappa$ of 1.0 represents perfect agreement, a $\kappa$ of 0.81 to 1.0 is excellent agreement, 0.61 to 0.80 is good agreement, 0.41 to 0.60 is moderate agreement, 0.21 to 0.40 is fair agreement and 0.0 to 0.20 represents slight agreement.

\section{Results}

The final pathological types of lymphomas were: nonHodgkin lymphomas in 102 patients (88.7\%) and Hodgkin lymphomas in 13 patients (11.3\%). The subtypes of the non-Hodgkin lymphomas were DLBCL in 69 patients $(60 \%)$, Burkitt lymphoma in 8 patients (7\%), follicular lymphoma 
Table 1. Inter-observer agreement of staging of lymphoma.

\begin{tabular}{ccccc}
\hline Stage & Observer 1 & Observer 2 & $\boldsymbol{K}$ & \multicolumn{2}{c}{ Percent agreement } \\
\hline Stage I & $14(12.1 \%)$ & $13(11.3 \%)$ & 0.93 & $96.4 \%$ \\
\hline Stage II & $18(15.7 \%)$ & $20(17.4 \%)$ & 0.90 & $94.8 \%$ \\
\hline Stage III & $33(28.7 \%)$ & $38(33.0 \%)$ & 0.89 & $94.6 \%$ \\
\hline Stage IV & $50(43.5 \%)$ & $44(38.3 \%)$ & 0.88 & $94.0 \%$ \\
\hline Overall & & & 0.90 & $94.9 \%$ \\
\hline
\end{tabular}

in 7 patients $(6.1 \%)$, small cell lymphoma in 5 patients (4.3\%), T-cell lymphoblastic lymphoma in 4 patients (3.5\%), anaplastic lymphoma in 4 patients (3.5\%), peripheral T-cell lymphoma in 2 patients (1.8\%), mantle cell lymphoma in 2 patients $(1.8 \%)$ and MALT lymphoma in only one patient (0.9\%). The subtypes of Hodgkin lymphomas were mixed cellularity type in 6 patients $(5.2 \%)$, nodular sclerosis type in 5 patients $(4.3 \%)$ and nodular lymphocytic predominance in 2 patients (1.7\%).

Table 1 shows inter-observer agreement with respect to staging of lymphomas. Figure 1 shows the different stages of lymphomas according to the Lugano classification. Stage I lymphoma was reported in 14 patients (12.1\%) by observer 1 and in 13 patients (11.3\%) by observer 2, with an excellent agreement $(k=0.93$ and percent of agreement was $96.4 \%)$. Stage II lymphoma was reported in 18 patients $(15.8 \%)$ by observer 1 and in 20 patients $(17.4 \%)$ by observer 2 , with an excellent agreement $(k=0.90$ and the percent of agreement was $94.8 \%)$. Stage III lymphoma was reported in 33 patients $(28.7 \%)$ by observer 1 and in 38 patients (33.0\%) by observer 2, with an excellent agreement $(k=0.89$ and the percent of agreement was $94.6 \%)$. Stage IV was reported in 50 patients $(43.5 \%)$ by observer 1 and in 44 patients $(38.3 \%)$ by observer 2 with an excellent agreement ( $k=0.88$ and the percent of agreement was $94 \%$ ). The overall inter-observer agreement of WBCT in staging of lymphoma was excellent ( $k=0.90$, percent agreement $=94.9 \%)$.

Table 2 shows the final treatment response assessment of lymphomas. Complete response (Figure 2) was reported in 13 patients $(11.3 \%)$ by observer 1 and in 15 patients (13.1\%) by observer 2 , with an excellent agreement $(k=0.87$ and the percent of agreement was 93.3\%). Partial response
(Figure 3) was detected in 27 patients $(23.5 \%)$ by observer 1 and in 26 patients $(22.6 \%)$ by observer 2 , with an excellent agreement $(k=0.96$ and the percent of agreement was 98.1\%). Stable disease (Figure 4) was reported in 30 patients $(26.1 \%)$ by observer 1 and in 27 patients $(23.5 \%)$ by observer 2 , with an excellent agreement $(k=0.90$ and percent of agreement was 95\%). Progressive disease (Figure 5) was reported in 33 patients $(28.7 \%)$ by observer 1 and in 35 patients (30.4\%) by observer 2, with an excellent agreement $(k=0.94$ and the percent of agreement was $97.1 \%)$. Both observer 1 and observer 2 lost 12 patients (10.4\%) to follow-up. The overall inter-observer agreement in response assessment was excellent $(k=0.91$, percent agreement $=95.8 \%$ ).

\section{Discussion}

In the present study, WBCT could detect nodal and extranodal spread of the disease in different regions of the body within a short examination time. There is an excellent inter-observer agreement of WBCT in staging of lymphoma $(k=0.90$, percent agreement $=94.9 \%)$ and treatment response assessment $(k=0.91$, percent agreement $=95.8 \%)$.

Pre- and posttreatment imaging for staging and treatment response assessment in lymphoma patients varies widely by center. The Lugano classification represents a consensus statement of clinical experts in lymphoma and is therefore expected to serve as a unified guideline for all physicians involved in lymphoma staging and response assessment. Contrast-enhanced CT will allow for more accurate measurements of node size if required for clinical trials and will help better discern adenopathy from the surrounding soft-tissue structures. The new response criteria should

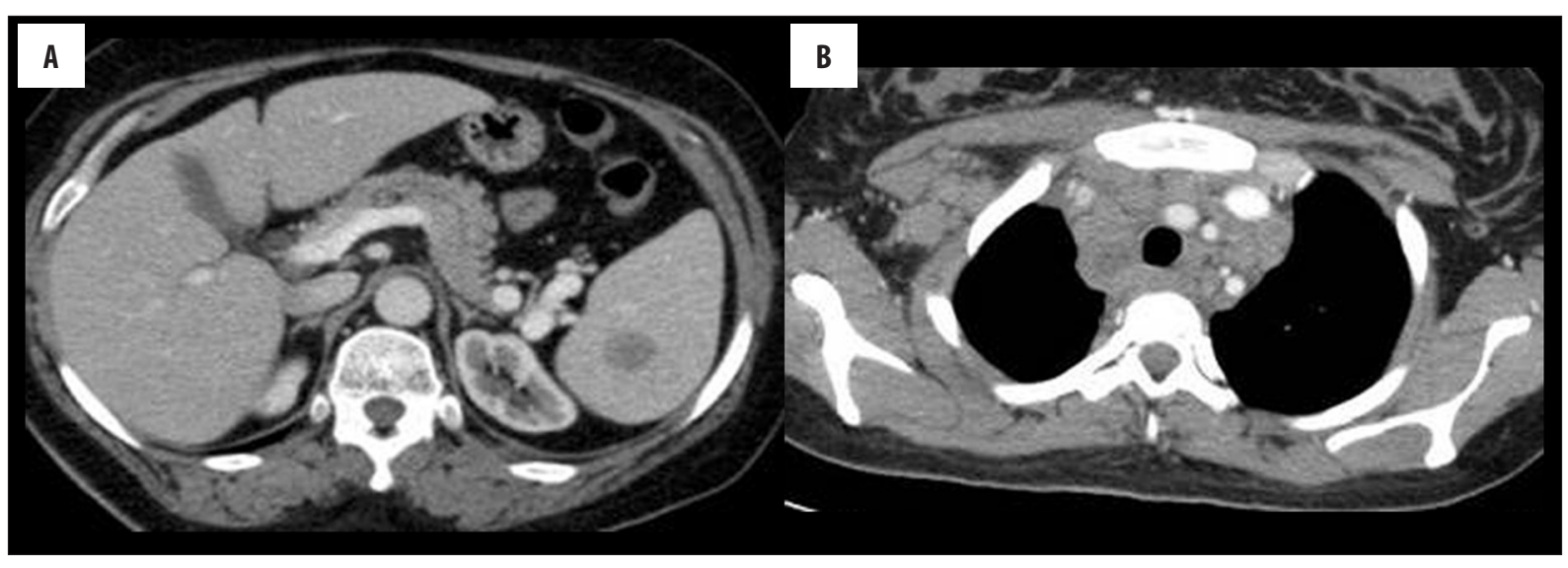




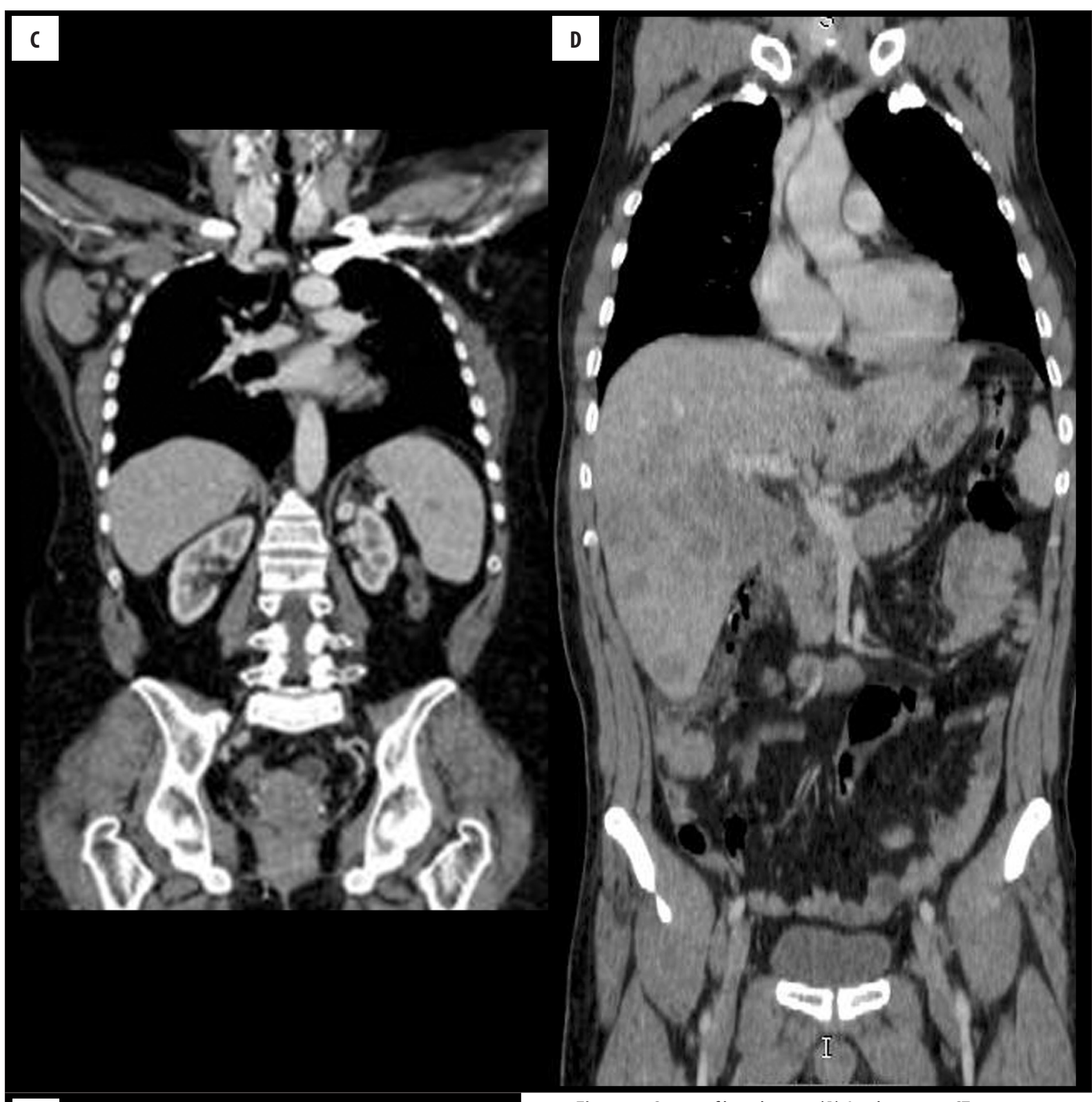

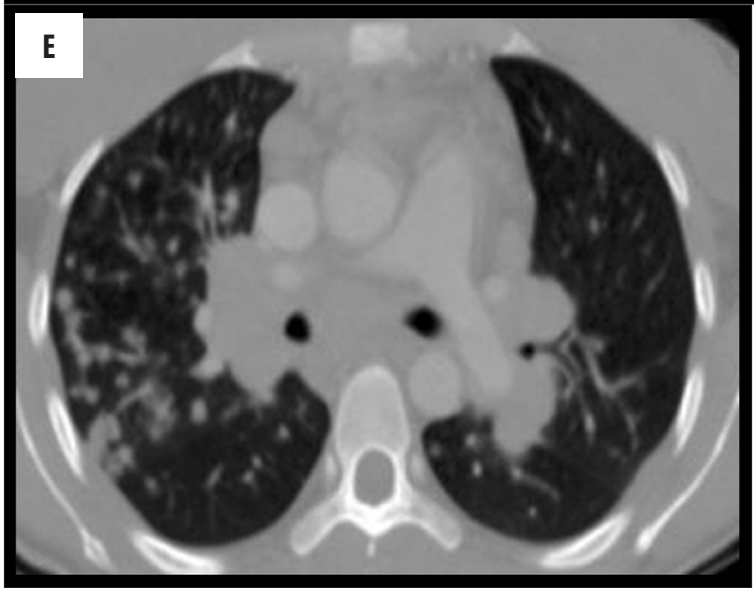

facilitate a simple assessment of treatment response in order to guide clinical management. Owing to these
Figure 1. Stages of lymphomas: (A) Axial, contrast CT scan of the abdomen shows a single splenic focal lesion (stage I). (B) Axial, contrast CT scan of the chest shows an amalgamated mass of multiple enlarged lymph nodes ipsilateral to the diaphragm (stage II). (C) Coronal WBCT shows enlarged axillary lymph nodes above the diaphragm and splenic focal lesions below the diaphragm (stage III). (D, E) Coronal WBCT shows multifocal hepatic lesions with multiple pulmonary nodules (stage IV).

criteria, radiologists have a renewed opportunity to guide clinical management based on imaging findings [4-6].

In this study, there was an excellent inter-observer agreement of WBCT in staging of nodal and extra-nodal of lymphoma. One study reported that staging of lymphomas is a vital pre-requisite to appropriate therapeutic management and prognostication [3]. Another study added that whole-body diffusion-weighted MR imaging and FDG 
Table 2. Inter-observer agreement of response assessment of lymphoma.

\begin{tabular}{lcccc}
\hline \multicolumn{1}{c}{ Stage } & Observer 1 & Observer 2 & $\boldsymbol{K}$ & Percent agreement \\
\hline Progressive disease & $33(28.7 \%)$ & $35(30.4 \%)$ & 0.94 & $97.1 \%$ \\
\hline Stable disease & $30(26.1 \%)$ & $27(23.5 \%)$ & 0.90 & $95.0 \%$ \\
\hline Partial response & $27(23.5 \%)$ & $26(22.6 \%)$ & 0.96 & $98.1 \%$ \\
\hline Complete response & $13(11.3 \%)$ & $15(13.1 \%)$ & 0.87 & $93.3 \%$ \\
\hline Lost follow up & $12(10.4 \%)$ & $12(10.4 \%)$ & & $95.8 \%$ \\
\hline Overall & & 0.91 & \\
\hline
\end{tabular}

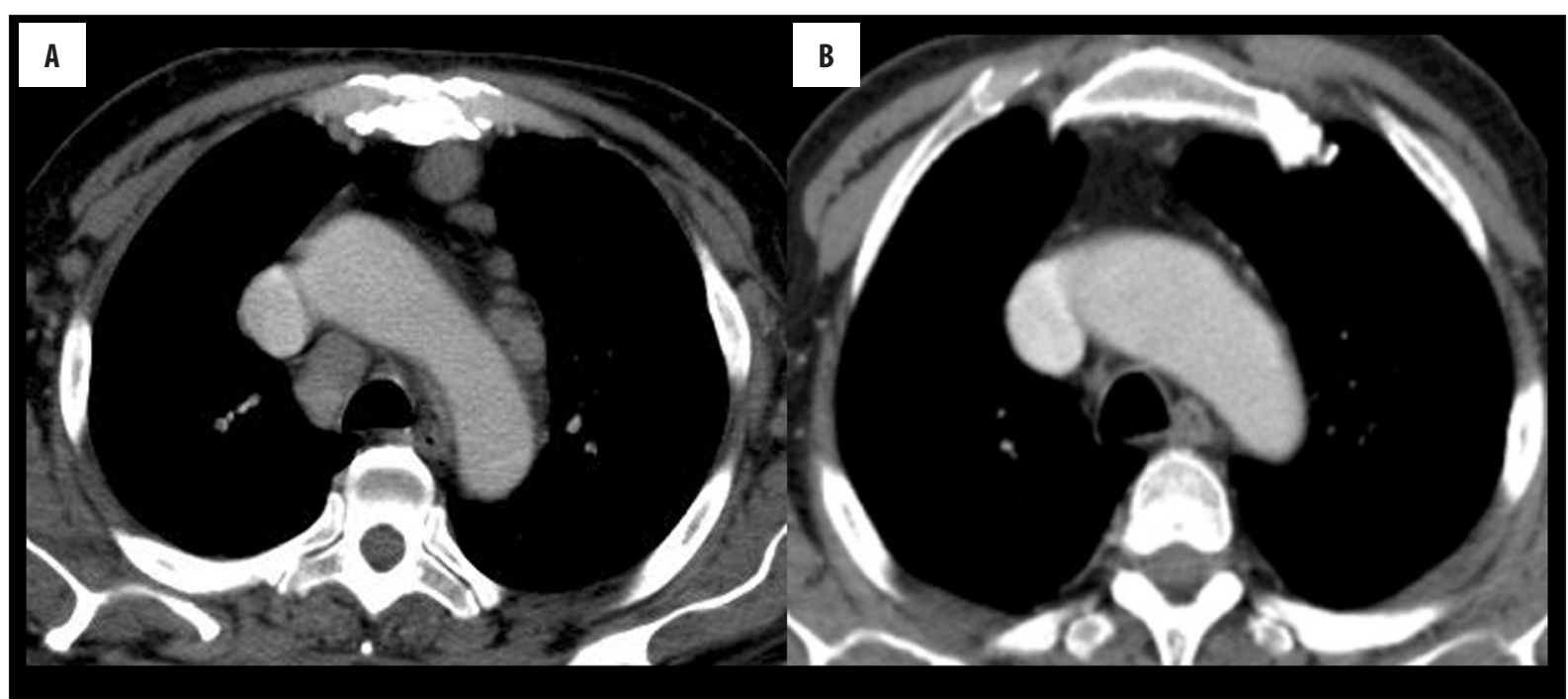

Figure 2. Complete response: (A) Axial, contrast CT before therapy shows enlarged mediastinal lymph nodes. (B) Axial, contrast CT scan after 4 cycles of chemotherapy shows complete disappearance of the target nodes.

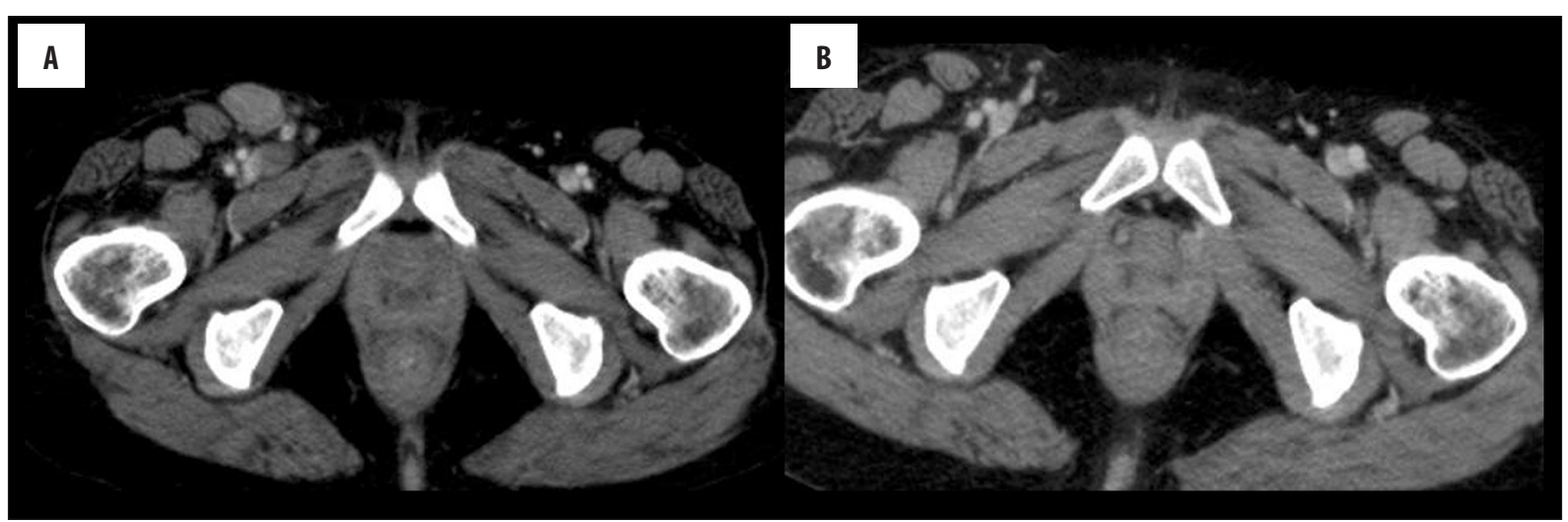

Figure 3. Partial response: (A) Axial CT shows enlarged inguinal lymph nodes. (B) Axial, contrast CT shows a regressive course with decreased sizes of inguinal lymph nodes.

PET/CT showed an overall good agreement $(K=0.796,95 \%$ IC $=0.651-0.941)$, especially in the evaluation of the nodal basins in NHL $(K=0.937,95 \%$ IC $=0.814-1)$ [27].

In this study, there was an excellent agreement between both reviewers with regard to treatment response assessment. In practice, posttreatment imaging in lymphoma patients varies widely by center and will probably remain variable despite the new consensus guidelines. Residual masses are seen on anatomic imaging in up to $80 \%$ of patients with HL and $40 \%$ of those with NHL [3]. One study reported that, in reference to the revised response criteria for malignant lymphomas, whole-body MR imaging and whole-body diffusion MR imaging showed a good agreement $(K=0.824,95 \%$ IC $=0.493-1)$ [27]. Another study added that, with regard to treatment response assessment, diffusion-weighted MR imaging agreed with 18F-FDG-PET/CT in $99 / 102$ of follow-up examinations (97.1\%), with a kappa value of $0.94(\mathrm{P}<.0001)$ [28]. 


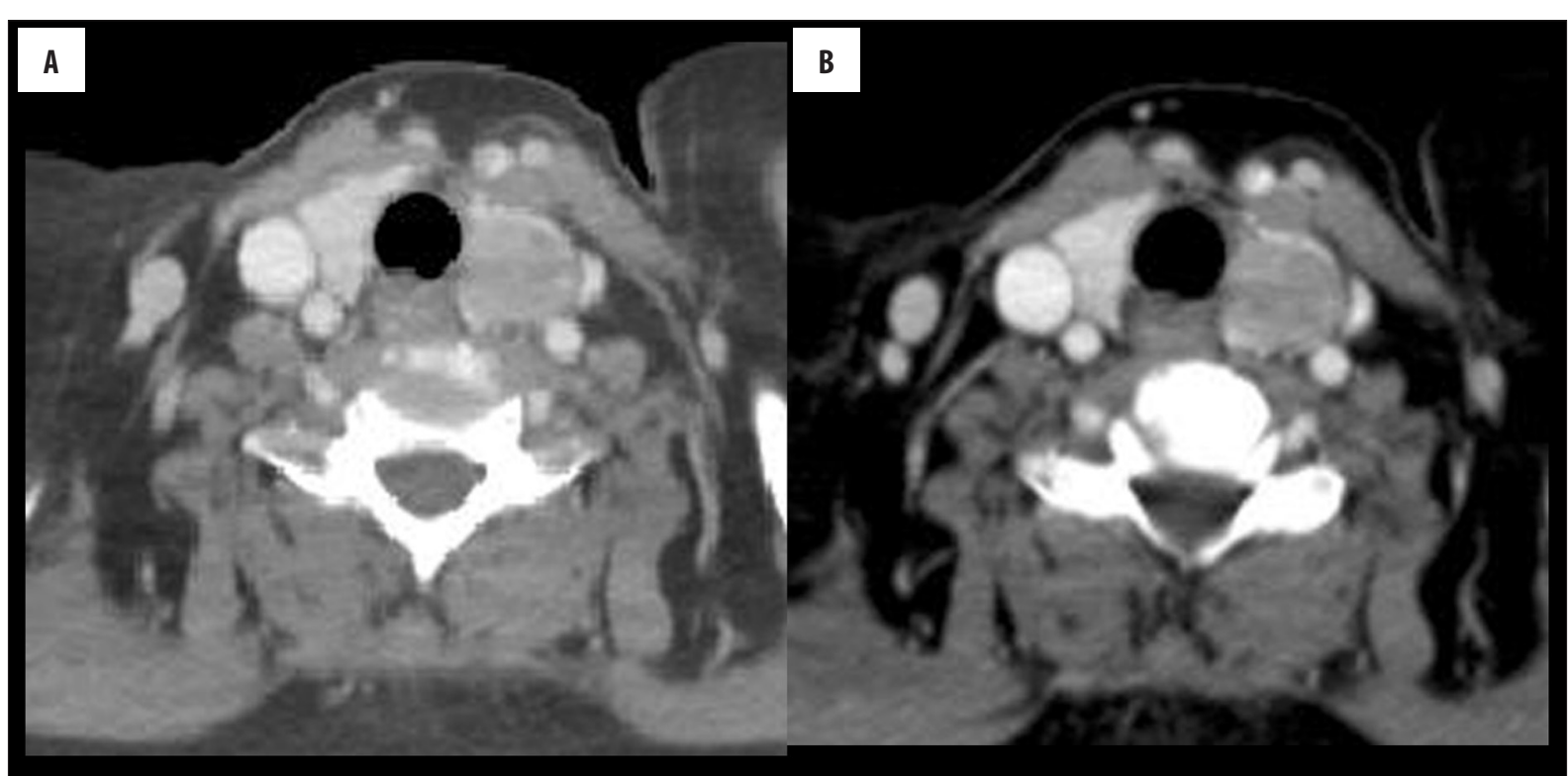

Figure 4. Stable Disease: (A) Axial, contrast CT scan shows focal lesions in the left thyroid lobe. (B) Axial, contrast CT scan after a complete course of chemotherapy shows stable appearance of the left thyroid focal lesion.

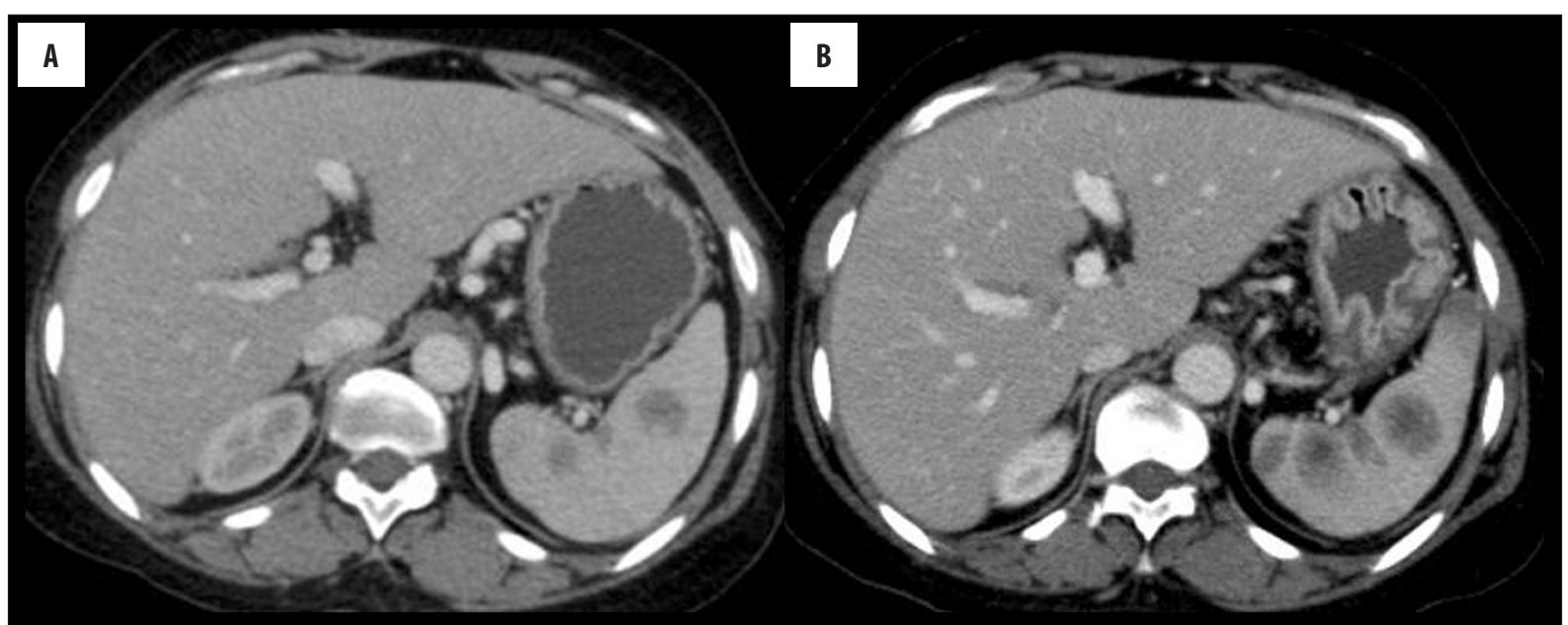

Figure 5. Progressive disease: (A) Axial CT scan shows few small focal lesions in the spleen. (B) Axial CT scan after a complete course of chemotherapy shows increased sizes and number of the splenic focal lesions.

CT is the most commonly used imaging modality for staging and response assessment in lymphoma because of its widespread availability, relatively low cost, rapid examination, very short scanning time and excellent resolution. The drawbacks of CT include patient exposure to ionizing radiation and administration of iodinated contrast medium $[29,30]$. An important disadvantage of CT is the use of ionizing radiation which may be associated with induction of secondary cancers in later life, administration of iodinated contrast agents that may cause adverse reactions, including contrast-induced nephrotoxicity. CT lacks functional information, which impedes identification of disease in normalsized nodes [14,15].

There are a few limitations of this study. First, this study was done using only WBCT and further studies with application of dual-energy CT [31-33], WB-PET-CT and whole-body diffusion MR imaging will improve the results. Second, this study was done in patients with lymphomas. Further studies in subtypes of non-Hodgkin lymphomas are recommended. Third, image analysis for response assessment was performed for one study after therapy; further studies with interim analyses after 2-3 cycles of chemotherapy are needed. Moreover, analysis of long-term surveillance of the tumor will enhance the role of imaging in these patients.

\section{Conclusions}

We concluded that WBCT is a reliable and reproducible imaging modality for staging and treatment response assessment in lymphomas according to the Lugano classification. 


\section{References:}

1. Siegel R, Miller KD, Jemal A: Cancer statistics, 2015. Cancer J Clin, 2015; 65: 5-29

2. Intlekofer AM, Younes A: Precision therapy for lymphoma - current state and future directions. Nat Rev Clin Oncol, 2014; 11: 585-96

3. Ansell SM: Non-Hodgkin lymphoma: Diagnosis and treatment. Mayo Clin Proc, 2015; 90: 1152-63

4. Johnson SA, Kumar A, Matasar MJ et al: Imaging for staging and response assessment in lymphoma. Radiology, 2015; 276: 323-38

5. Cheson BD: Staging and response assessment in lymphomas: The new Lugano classification. Clin Oncol, 2015; 4: 5

6. Cheson BD, Fisher RI, Barrington SF et al: Recommendations for initial evaluation, staging, and response assessment of Hodgkin and non-Hodgkin lymphoma: The Lugano classification. J Clin Oncol, 2014; 32: 3059-68

7. El-Galaly TC, Hutchings M: Imaging of non-Hodgkin lymphomas: Diagnosis and response-adapted strategies. Cancer Treat Res, 2015; 165: $125-46$

8. Barrington SF, Mikhaeel NG, Kostakoglu L et al: Role of imaging in the staging and response assessment of lymphoma: Consensus of the International Conference on Malignant Lymphomas Imaging Working Group. J Clin Oncol, 2014; 32: 3048-58

9. Dupas B, Augeul-Meunier K, Frampas E et al: Staging and monitoring in the treatment of lymphomas. Diagn Interv Imaging, 2013; 94: in the tre

10. Razek AA, Tawfik AM, Elsorogy LG, Soliman NY: Perfusion CT of head and neck cancer. Eur J Radiol, 2014; 83: 537-44

11. Wang X: PET/CT: Appropriate application in lymphoma. Chin Clin Oncol, 2015; 4: 4

12. Moskowitz CH, Schöder H: Current status of the role of PET imaging in diffuse large B-cell lymphoma. Semin Hematol, 2015; 52: 138-42

13. Papamichael DC, Pianou NK, Chatzipavlidou V: The role of positron emission tomography in in lymphoma diagnosis and treatment. Hell J Nucl Med, 2015; 18: 173-79

14. Toledano-Massiah S, Luciani A, Itti E et al: Whole-body diffusionweighted imaging in Hodgkin Lymphoma and diffuse large B-cell lymphoma. Radiographics, 2015; 35: 747-64

15. Abdel Razek AA, Soliman NY, Elkhamary S et al: Role of diffusionweighted MR imaging in cervical lymphadenopathy. Eur Radiol, 2006; 16: 1468-77

16. Lin C, Luciani A, Itti E et al: Whole-body diffusion magnetic resonance imaging in the assessment of lymphoma. Cancer Imaging, 2012; 12: 403-8

17. Razek AA: Diffusion-weighted magnetic resonance imaging of head and neck. J Comput Assist Tomogr, 2010; 34: 808-15

18. Hagtvedt T, Seierstad T, Lund KV et al: Diffusion-weighted MRI compared to FDG PET/CT for assessment of early treatment response in lymphoma. Acta Radiol, 2015; 56: 152-58
19. Abdel Razek AA, Kandeel AY, Soliman N et al: Role of diffusionweighted echo-planar MR imaging in differentiation of residual or recurrent head and neck tumors and posttreatment changes. Am J Neuroradiol, 2007; 28: 1146-52

20. Heacock L, Weissbrot J, Raad R et al: PET/MRI for the evaluation of patients with lymphoma: initial observations. Am J Roentgenol, 2015; 204: 842-48

21. Razek AA, Ezzat A, Azmy E, Tharwat N: Role of whole-body 64-slice multidetector computed tomography in treatment planning for multiple myeloma. Radiol Med, 2013; 118: 799-805

22. Shannon L, Peachey T, Skipper $N$ et al: Comparison of clinically suspected injuries with injuries detected at whole-body CT in suspected multi-trauma victims. Clin Radio, 2015; 70: 1205-11

23. Adams HJ, Kwee TC, Vermoolen MA et al: Whole-body MRI vs. CT for staging lymphoma: Patient experience. Eur J Radiol, 2014; 83: $163-66$

24. Fueger BJ, Yeom K, Czernin J et al: Comparison of CT, PET, and PET/ CT for staging of patients with indolent non-Hodgkin's lymphoma Mol Imaging Biol, 2009; 11: 269-74

25. Brennan DD, Gleeson T, Coate LE et al: A comparison of whole-body MRI and CT for the staging of lymphoma. Am J Roentgenol, 2005; 185: 711-16

26. Armitage JO: Staging non-Hodgkin lymphoma. Cancer J Clin, 2005; 55: $368-76$

27. Maggialetti N, Ferrari C, Minoia C et al: Role of WB-MR/DWIBS compared to (18)F-FDG PET/CT in the therapy response assessment of lymphoma. Radiol Med, 2016; 121: 132-43

28. Mayerhoefer ME, Karanikas G, Kletter $\mathrm{K}$ et al: Evaluation of diffusion-weighted magnetic resonance imaging for follow-up and treatment response assessment of lymphoma: Results of an 18F-FDG$\mathrm{PET} / \mathrm{CT}$-controlled prospective study in 64 patients. Clin Cancer Res, 2015; 21: 2506-13

29. Meyer M, Klein SA, Brix G et al: Whole-body CT for lymphoma staging: Feasibility of halving radiation dose and risk by iterative image reconstruction. Eur J Radiol, 2014; 83: 315-21

30. Guttikonda R, Herts BR, Dong F et al: Estimated radiation exposure and cancer risk from CT and PET/CT scans in patients with lymphoma. Eur J Radiol, 2014; 83: 1011-15

31. Tawfik AM, Kerl JM, Razek AA et al: Image quality and radiation dose of dual-energy CT of the head and neck compared with a standard 120-kVp acquisition. Am J Neuroradiol, 201 1; 32: 1994-99

32. Tawfik AM, Razek AA, Kerl JM et al: Comparison of dualenergy CT-derived iodine content and iodine overlay of normal, inflammatory and metastatic squamous cell carcinoma cervical lymph nodes. Eur Radiol, 2014; 24: 574-80

33. Tawfik AM, Razek AA, Elhawary G, Batouty NM: Effect of increasing the sampling interval to 2 seconds on the radiation dose and accuracy of CT perfusion of the head and neck. J Comput Assist Tomogr, 2014; 38: 469-73 